Original article

\title{
A chair-side plasma treatment system for rapidly enhancing the surface hydrophilicity of titanium dental implants in clinical operations
}

\author{
Yuqing Dong ${ }^{1,2)}$, Li Long ${ }^{3)}$, Peng Zhang ${ }^{1,2)}$, Deping $\mathrm{Yu}^{1,2)}$, Yana Wen ${ }^{1)}$, Zheng Zheng ${ }^{3)}$, Jie $\mathrm{Wu}^{1)}$, and Wenchuan Chen ${ }^{3)}$ \\ School of Mechanical Engineering, Sichuan University, Chengdu, P. R. China \\ ${ }^{2)}$ Yibin Research Institute, Sichuan University, Yibin, P. R. China \\ State Key Laboratory of Oral Diseases and National Clinical Research Center for Oral Diseases, West China Hospital of Stomatology, Sichuan University, Chengdu, P. R. China
}

\begin{abstract}
Purpose: In order to promote osseointegration and shorten the healing time after dental implant operations, this study was conducted to develop a chair-side plasma treatment system in which Ti implants were used as a coaxial internal electrode to rapidly enhance their surface hydrophilicity. Methods: Surface hydrophilicity was evaluated by measurement of the water contact angle and the defined wetting time. Changes in temperature and chemical composition were analyzed using infrared thermal imaging and X-ray photoelectron spectroscopy (XPS), respectively. The biocompatibility of the treated implants was examined in an animal experiment.

Results: A marked improvement of hydrophilicity was demonstrated by a decrease in the water contact angle of the treated implant to $0^{\circ}$ and wetting of the whole surface within $3 \mathrm{~s}$ of water contact. The Ti implant hydrophilization mechanism was explained as a decrease in the degree of hydrocarbon contamination. The surface temperature of the treated implant was close to that of the human body, and good osseointegration was observed in the in vivo experiment.

Conclusion: The plasma treatment system developed here is a promising chair-side procedure for rapidly enhancing the surface hydrophilicity of Ti implants in clinical operations without any need to consider the degradation of hydrophilicity caused by long-term storage.
\end{abstract}

Keywords; chair-side procedure, cold plasma, hydrophilic treatment, titanium dental implant

\section{Introduction}

In recent years, dental implants have been widely used to solve problems associated with missing teeth, facilitating a repairing effect equivalent to that of real teeth $[1,2]$. Biocompatibility, chemical stability and resistance, favorable mechanical properties and reasonable machinability are all important factors for dental implant materials, but some metals such as stainless steel may cause adverse tissue reactions leading to a low success rate due to their poor corrosion resistance, whereas ceramic materials such as zirconia, despite their excellent biocompatibility and mechanical properties, are difficult to machine. Up to now, titanium and its alloys have been the most widespread and successfully used materials for dental implants because of their biocompatibility, corrosion resistance, light weight, durability, and ease of machining, possessing a modulus of elasticity similar to that of bone [3]. However, due to the bioinert nature of titanium, normal implants without hydrophilicity barely combine with osteoblasts and proteins, causing poor osseointegration [4-6], whereas hydrophilic implants can significantly promote osseointegration, shorten healing time, and increase the success rate of implant operations [7-9]. Currently, blasting and acid-etching [9] and coating technology [10] have been applied in

Correspondence to Dr. Peng Zhang, School of Mechanical Engineering, Sichuan University, No.24, South Section 1, 1st ring road, Chengdu 610065, Sichuan, P. R. China

Fax: +86-28-85460940 E-mail: pengzhang@scu.edu.cn

Color figures can be viewed in the online issue at J-STAGE.

doi.org/10.2334/josnusd.21-0090

DN/JST.JSTAGE/josnusd/21-0090 factories to improve the surface hydrophilicity of implants by changing the surface topography and introducing bioactive coating, respectively. However, these methods are complex and expensive. More importantly, maintaining the implant surface hydrophilicity is still challenging because it degrades rapidly with increased storage time. If hydrophilic treatment is undertaken solely in factories, a proportion of implants would lose their hydrophilicity completely by the time they are used in clinical operations [11]. Therefore, use of a simpler treatment method as a chair-side procedure is urgently needed for addressing this issue of pre-acquired hydrophilicity degrading with storage time.

Ultraviolet irradiation [7] and plasma treatment [12] are typical examples of simpler treatment methods that do not alter the surface topography of implants. However, ultraviolet irradiation usually requires a long irradiation time, making hydrophilic treatment in clinical operations impracticable. In comparison, as an electrically neutral and ionized gas close to room temperature, cold atmospheric plasma (CAP) is a more rapid treatment modality, and has been widely used for surface modification of materials such as aluminum alloys [13], stainless steel [14], titanium alloys [15] and polymers [16] to enhance their surface hydrophilicity and adsorption properties. Furthermore, as a modification technology that modifies the surface to only a few nanometers, Chu [17] has demonstrated that CAP can alter the surface biological properties of biomaterials without any change in bulk properties such as strength and inertness. Duske and Lee et al. $[12,18,19]$ have utilized the plasma jet technique to treat $\mathrm{Ti}$ discs for about 2 min and proved that this yields not only excellent surface hydrophilicity but also superior antibacterial ability. More importantly, by culturing MG-63 human osteoblasts in vitro, Duske et al. [18] have demonstrated that the spreading behavior of the cells on treated titanium discs is dramatically improved, with an increase in the cell-occupied area of up to $57-86 \%$ in comparison with untreated Ti discs. In addition, Naujokat and Guastaldi et al. [8,20], employing miniature pigs and beagle dogs, respectively, have proved that Ti implants after plasma jet treatment can promote bone formation in vivo, characterized by a slightly higher degree of bone-to-implant contact (BIC) and a significantly higher level of bone area fraction occupancy (BAFO) relative to untreated implants. Hence plasma treatment appears to be a promising way to convert normal implants to hydrophilic implants in clinical operations, because improved hydrophilicity can be obtained in a short time without any need to consider the degradation of hydrophilicity caused by long-term storage. However, due to the screw shape of the implant surface, as shown in Fig. 1(b), plasma in the form of a jet makes it difficult to treat a whole implant surface simultaneously, requiring practitioners to keep adjusting the relative position of the implant to the plasma jet manually. Such a relatively complex and uncontrollable treatment process would result in heterogeneity of the treatment effect. Consequently, treatment with a plasma jet is not the most ideal modality for use as a chair-side procedure in clinical operations. Taking into account the high conductivity of titanium alloy, a Ti implant can be employed directly as a coaxial internal electrode to generate cold plasma, submerging the whole implant and facilitating rapid hydrophilic treatment. Here, the dielectric barrier discharge (DBD) needs to be adopted by inserting dielectric materials such as quartz between the internal and external electrodes to ensure a uniform discharge.

This report describes the development of a coaxial DBD plasma treatment system in which a Ti dental implant is used as an internal electrode to 
(a)

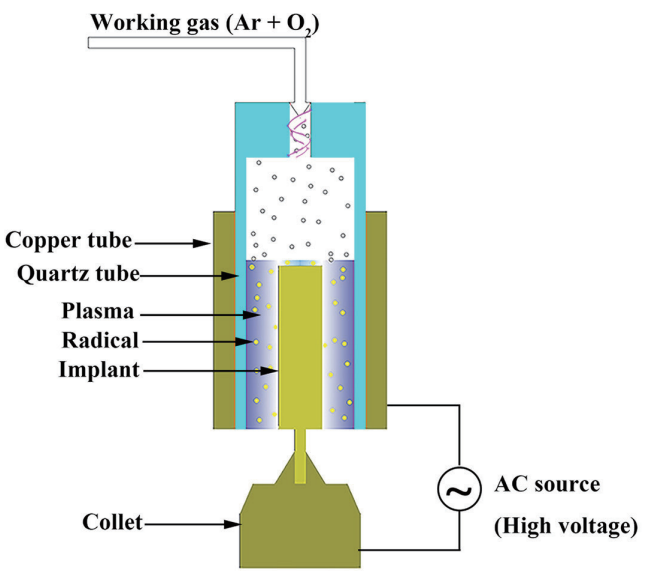

(b)

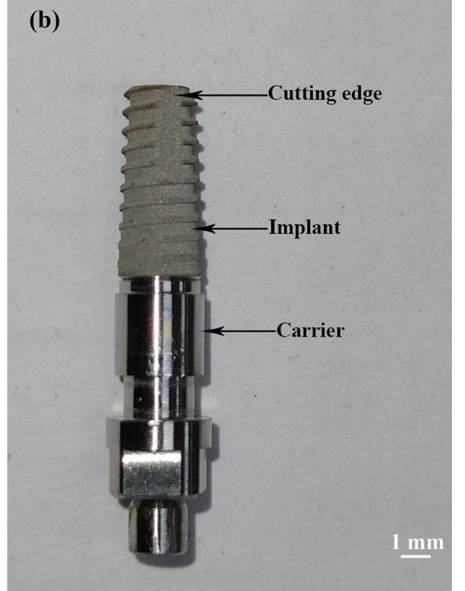

Fig. 1 Schematic diagram of the coaxial DBD plasma treatment system (a), and a photo of the dental implant used in this work (b)

generate the CAP. Argon was used as the primary working gas to generate the CAP as it is low-cost and easy to ionize; a slight amount of oxygen was also added to produce oxygen-containing groups, which can further enhance the hydrophilicity and the antibacterial properties of the Ti implant. The wettability of the treated implants was then characterized by measuring the water contact angle and the defined wetting time. Clinical effectiveness and safety were verified by detecting the osseointegration state. Moreover, the chemical composition of the implant surface was analyzed by X-ray photoelectron spectroscopy (XPS) to further explore the hydrophilization mechanism of the plasma treatment. The surface temperature of the treated implants was also measured by infrared thermal imaging to ensure clinical safety. Finally, it was demonstrated that the coaxial DBD plasma treatment system was able to rapidly enhance the surface hydrophilicity of the Ti dental implants at a temperature close to that of the human body.

\section{Materials and Methods}

\section{Plasma treatment system}

Figure 1(a) shows a schematic diagram of the coaxial DBD plasma treatment system, including the AC source, collet, implant, quartz tube, copper tube, and working gas. By adjusting the input current $(220 \mathrm{~V}, 50 \mathrm{~Hz})$, the $\mathrm{AC}$ source was able to provide an alternating voltage of several kilovolts with a power of less than $50 \mathrm{~W}$ to ionize the working gas at room temperature.

In order to respond to the rotary shape feature of the Ti implant, the latter was used as the coaxial internal electrode to conduct the DBD. The generated CAP was able to treat the whole implant surface simultaneously. As shown in Fig. 1(a), the implant was mounted on a metal collet, which was fixed by a fastening bolt and connected to the output of the AC source. The implant and the collet both served as the internal electrode. A quartz tube with an internal diameter of $8 \mathrm{~mm}$ and an external diameter of $10 \mathrm{~mm}$ acted as a dielectric barrier to avoid any arc discharge that could damage the implant surface. As the external electrode, a copper tube with an internal diameter of $10 \mathrm{~mm}$ and an external diameter of $12 \mathrm{~mm}$ covered the quartz tube and was connected to the output of the AC source. As the implant, collet, quartz tube, and copper tube were coaxial, the generated CAP was distributed uniformly in the space between the implant and the quartz tube, and the whole implant was submerged.

The system used argon as the primary gas and oxygen as the additional gas, the flowrates being precisely controlled by two mass flow controllers (MFC). Generally, the introduction of the oxygen-containing polar groups not only enhances the surface hydrophilicity of the implant but also enhances its antibacterial properties [15,21,22]. The gas mixture accessed the quartz tube via a helical route to match the screw shape of the Ti implant and to maximize the area of contact between the reactive radicals and the implant surface. Table 1 summarizes the basic parameters employed in this study.

\section{Materials and hydrophilization procedures}

The commercial Ti implant with a blast surface was used for this study (grade IV, WeiHai Wego Jericom Biomaterials Co., Ltd., Weihai, P. R.
China). The mechanical properties of the implant were ascertained from the product description (modulus $104 \mathrm{GPa}$, ultimate tensile strength 550 $\mathrm{MPa}$, yield strength $483 \mathrm{MPa}$, elongation $15 \%$, density $4.5 \mathrm{~g} / \mathrm{cc}$ ). The shape of the implant is shown in Fig. 1(b). Two flat cutting edges at the tip were used to detect the water contact angle, and a cylindric carrier at the end of the implant allowed it to be clamped by the collet. The length and diameter of the implant were $7 \mathrm{~mm}$ and $3 \mathrm{~mm}$, respectively.

To conduct the plasma treatment, the following operation steps were carried out. First, the implant was placed on the collet using sterilized hemostatic forceps, the collet was tightened, and the treatment chamber was closed manually. Next, using the touch panel of the system, the treatment parameters were set, including the treatment time (7-75 s), the current of the AC source (0.13-0.21 A), the argon flow rate (1-5 SLM), and the oxygen content $(0-2 \%)$. Finally, the power of the AC source was turned on, and the implant was submerged in the generated CAP until the desired treatment time had elapsed. After the treatment, the chamber was opened and the collet was released manually. The implant was then removed using sterilized hemostatic forceps for further measurements or procedures.

\section{Detection and analysis}

Wetting property

To determine the change in the implant surface hydrophilicity after plasma treatment, the water contact angles of the same implant before and after the treatment were measured using a contact angle goniometer (PT-705B, Dong Guan Precise Test Equipment Co., Ltd., Dongguan, P. R. China). The measurements were conducted immediately after the plasma treatment, allowing the effect of storage time on the results to be ignored. Due to the screw shape of the implant surface, the measurement position was selected in a small flat area of the cutting edge, and a water drop volume of only $0.5 \mu \mathrm{L}$ was used.

Also, as shown in Fig. 2, hydrophilic implants are able to draw water and the whole implant surface becomes wet immediately when the tip touches the water surface, whereas untreated implants do not exhibit this property. Therefore, the period from touching the water surface until complete wetting was achieved was defined as the surface wetting time of the implant, which allows the hydrophilicity of the implant to be further evaluated using different treatment parameters. A total of 23 specimens were divided into five experimental groups, corresponding to five treatment parameter variables (treatment time, AC source current, argon flow rate, oxygen content, storage time). Except for the group in which the treatment time was varied (seven specimens), each of the other three groups included five specimens, and each experimental group had one common basic treatment parameter set, as listed in Table 1. Video recordings of the surface wetting process of each implant were taken. The surface wetting time was then calculated by counting the video frames ( 30 frames being equivalent to $1 \mathrm{~s}$ ). The videos were taken immediately after the plasma treatment, allowing the effect of storage time on the results to be ignored. Each experiment using different treatment parameters was repeated 6 times $(n=6)$ and a total of 138 specimens were assessed, the data being expressed as means and standard deviations. 

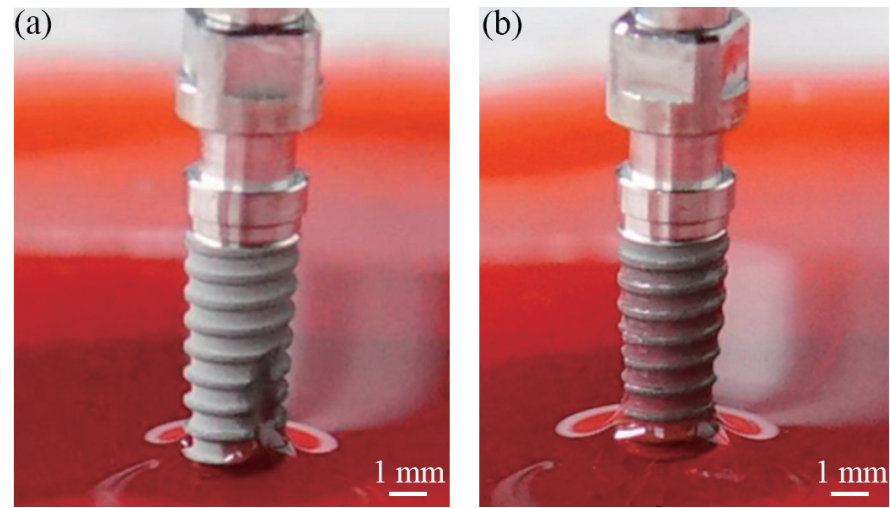

Fig. 2 Comparison of the wetting capabilities of untreated (a) and treated (b) Ti implants

Table 1 Basic treatment parameters employed in this work

\begin{tabular}{ll}
\hline Parameter & Value \\
\hline Treatment time & $30 \mathrm{~s}$ \\
Input current of AC source & $0.15 \mathrm{~A}$ \\
Flow rate of argon & $5.01 \times 10^{-5} \mathrm{~m}^{3} / \mathrm{s}(3 \mathrm{SLM})$ \\
Content of oxygen & $2.51 \times 10^{-7} \mathrm{~m}^{3} / \mathrm{s}(0.5 \%, 15 \mathrm{SCCM})$ \\
\hline
\end{tabular}

\section{Surface temperature}

To ensure the safety of each treated Ti implant for use in an immediate implant operation, the surface temperature of the treated implant was measured using an infrared thermal camera (R300W2 R15, NEC Avio Infrared Technologies Co., Ltd., Tokyo, Japan). Corresponding to the four treatment variables (treatment time, AC source current, argon flow rate, oxygen content), four experiment groups containing a total of 19 treatment parameter setups were designed, but to reduce the cost only one implant was repeatedly treated and subjected to measurements. The temperature distribution of the implant surface was recorded as a thermal image immediately after the plasma treatment, and the maximum temperature is displayed in Fig. 3. When the implant had cooled to room temperature, the next treatment and measurement were conducted. Each experiment using different treatment parameters was repeated 6 times $(n=6)$, and the data were expressed as means and standard deviations.

\section{Surface chemical composition}

To further explore the hydrophilization mechanism of the plasma treatment, changes in the chemical composition of the implant surface were analyzed by X-ray photoelectron spectroscopy (XPS, ESCALAB 250Xi, Thermo Fisher Scientific, Waltham, MA, USA). Four treated implants with different treatment times and one untreated implant were placed in sterilized sample bags and then subjected to XPS measurement, to reduce the effect of storage on the results as far as possible. Here the detection depth did not exceed $10 \mathrm{~nm}$. The survey spectra of the implant surface and the corresponding high-resolution spectra of $\mathrm{C} 1 \mathrm{~s}, \mathrm{O} 1 \mathrm{~s}$ and $\mathrm{Ti} 2 \mathrm{p}$ were analyzed using the Thermo Scientific Advantage software package (Thermo Fisher Scientific).

\section{In vivo animal experiments}

The research protocol was approved by the State Key Laboratory of Oral Diseases \& National Clinical Research Center for Oral Diseases (WCHSIRB-D-2021-003) conducted under the ARRIVE (Animals in Research: Reporting In Vivo Experiments) guidelines. All experiments were carried out in accordance with institutional and national animal care guidelines. A 1-year-old beagle dog weighing approximately $10 \mathrm{~kg}$ was used in this work. For surgery, the dog was anesthetized with Sumianxin II $(0.04 \mathrm{~mL} /$ $\mathrm{kg})$ and Zoletil $(0.3 \mathrm{mg} / \mathrm{kg})$. The mandibular second to third premolars were then extracted. After 3 months of healing, one treated Ti implant with the treatment parameters listed in Table 1 and one untreated implant were placed in the mandible, respectively. All drilling procedures were performed using the standard protocol. After 4 weeks of implantation, the dog was euthanatized with a lethal injection of $30 \% \mathrm{w} / \mathrm{v}$ chloral hydrate

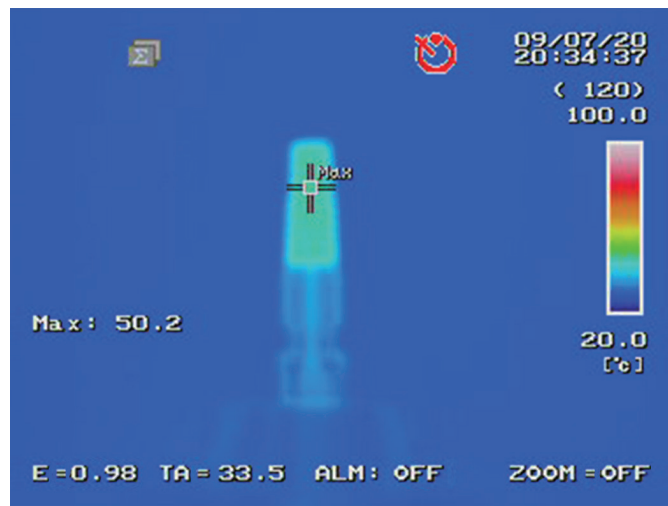

Fig. 3 A thermal image of the Ti implant after treatment using the parameters listed in Table 1

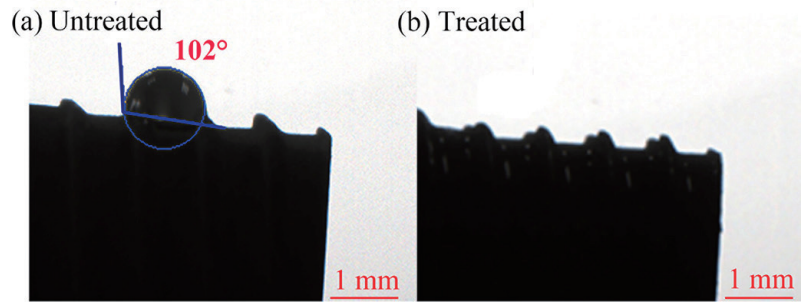

Fig. 4 Change in the water contact angle of the Ti implant after treatment using the parameters listed in Table 1

solution, and the mandible specimen was fixed in $4 \%$ paraformaldehyde. To detect the newly formed bone around the implant, the sample was scanned using micro-CT (Scano Medical AG, Basserdorf, Switzerland). The scanning parameters were set at $90 \mathrm{kV}$ and $200 \mu \mathrm{A}$ with an exposure time of $500 \mathrm{~ms}$ and a resolution of $15 \mu \mathrm{m}$. Cross-sectional images were then taken (Amira 6, Thermo Fisher Scientific).

\section{Statistical analysis}

To evaluate the statistical power and effect size, power analysis was conducted using the $G^{*}$ Power version 3.1.9.2 software package (program, concept and design by Franz Faul, Kiel University, Germany. Freely available Windows application software) [23]. The type of power analysis was selected as sensitivity, with a significance level $\alpha$ of 0.05 and a statistical power $(1-\beta)$ of 0.8 . With treatment time as the variable, the number of groups was 7 , the sample size was $42(n=6)$, and the calculated effect size was 0.62 ; when other treatment parameters were used as the variable, the number of groups was 5 , the sample size was $30(n=6)$, and the calculated effect size was 0.69 .

All statistical analyses were conducted using Origin 2019b software (OriginLab Corporation, Northampton, MA, USA). The results of Levene's test showed that the data collected did not satisfy homogeneity of variance $(P<0.05)$. To evaluate the effect of treatment time, the AC source current, the argon flow rate, the oxygen content, and the storage time on the wetting time and surface temperature, Kruskal-Wallis ANOVA (analysis of variance), which is a non-parametric test method, was performed. Dunn's test was used as a post-hoc analysis for multiple comparisons. $P<0.05$ was set as the level of significance.

\section{Results}

\section{Wetting property}

Figure 4 shows the changes in the water contact angle for the untreated and treated implants. It can be seen that the water contact angle of the untreated implant exceeded $102^{\circ}$, whereas the treated implant became wet instantly at the moment of contact with the water drop. Because the water was rapidly absorbed onto the threaded implant surface, the very small water contact angle could not be detected. Thus it was deduced that the Ti implant had obtained great surface hydrophilicity as a result of the plasma treatment and that the water contact angle was almost $0^{\circ}$. Han et al. [15] reported that the water contact angle of a Ti disc surface was also close to $0^{\circ}$ after hydrophilic treatment lasting $20 \mathrm{~s}$ using an atmospheric plasma jet. Consequently, irrespective of the Ti disc or Ti implant, it seems difficult to 

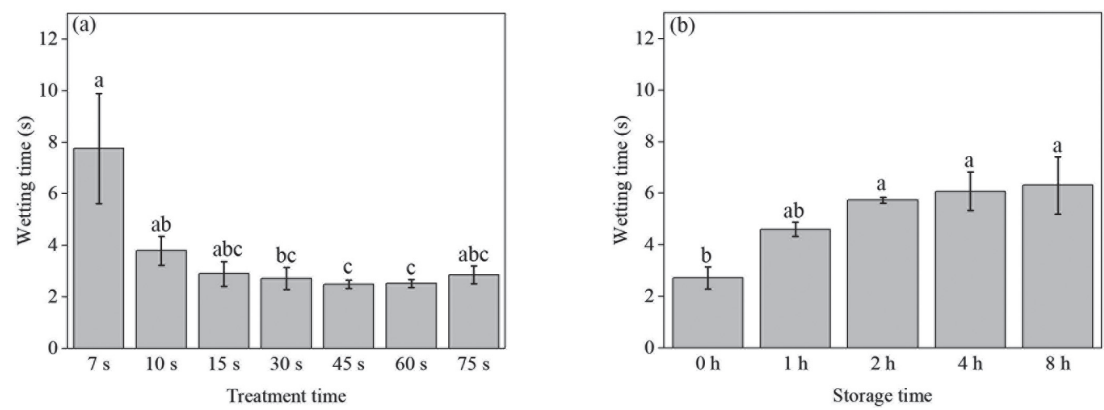

Fig. 5 Dependence of implant wetting time on the treatment time using the coaxial DBD plasma treatment system (a) and the time of storage in air (b). Error bars indicate standard deviations. Same letters indicate non-significant differences $(P>0.05$; Dunn's test).
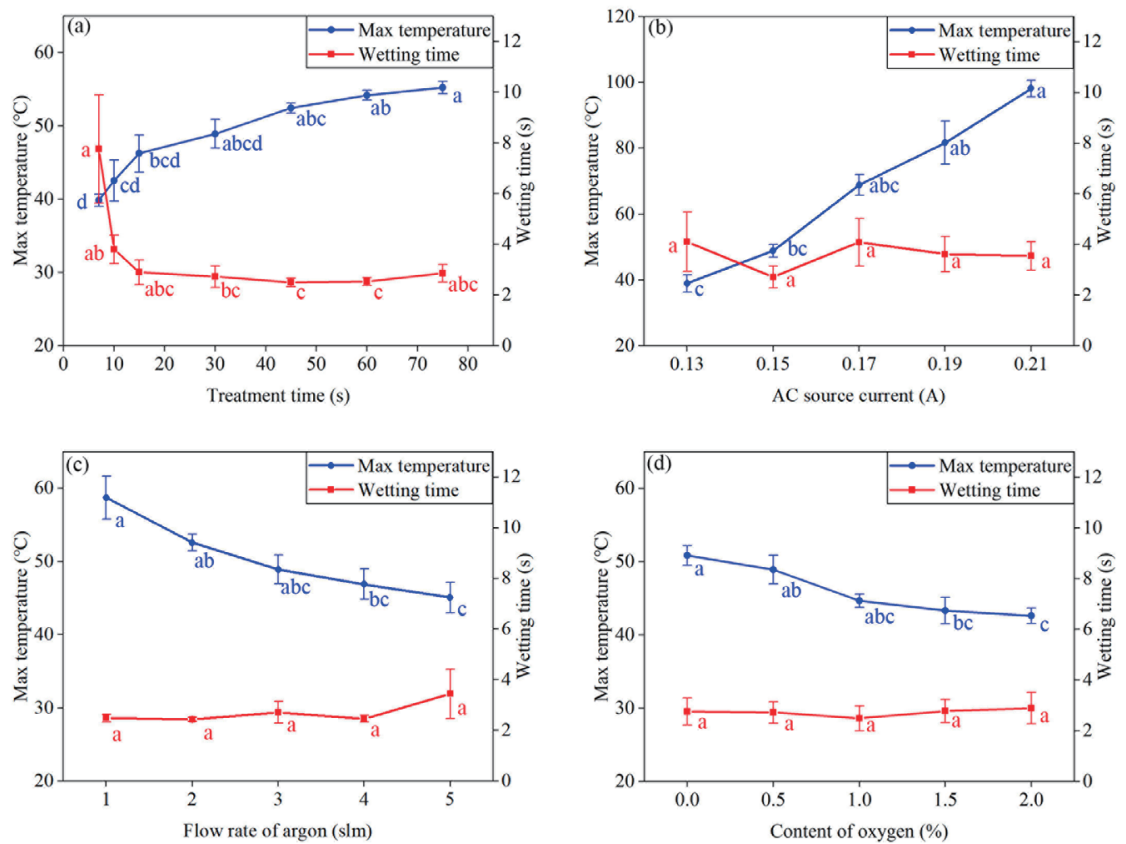

Fig. 6 Variations in the maximum temperature and wetting time of the treated implants with the treatment time (a), AC source curren (b), argon flow rate (c), and oxygen content (d). Except for the corresponding varying parameter, others parameters were set as the basic parameters listed in Table 1. Error bars indicate standard deviations. Same letters indicate non-significant differences $(P>0.05$; Dunn's test).

distinguish the effects of treatment merely by measuring the water contact angle when different treatment parameters are employed.

To clarify the effectiveness of the coaxial DBD plasma treatment for rendering hydrophilicity, the surface wetting time was employed as a quantitative variable. Figure 5(a) shows the variation in the surface wetting time of implants with treatment time when other treatment parameters were set as basic parameters listed in Table 1. It can be seen that the implant was completely wetted as long as the treatment time was as long as $7 \mathrm{~s}$, but a wetting time of $7.8 \pm 2.1 \mathrm{~s}$ was required. When the treatment time was increased to $30 \mathrm{~s}$, the wetting time required decreased to $2.7 \pm 0.4 \mathrm{~s}$, which was significantly shorter than that after treatment for $7 \mathrm{~s}(P=0.029)$. However, no significant differences in the wetting time was observed when the treatment time exceeded $30 \mathrm{~s}$. In other words, any further increase in the treatment time did not significantly reduce the wetting time. This suggests that the surface hydrophilicity of the implant appeared to peak after coaxial DBD plasma treatment for $30 \mathrm{~s}$. Certainly, the hydrophilicity of a Ti implant surface is known to inevitably degrade under general storage exposed to air [11]. As shown in Fig. 5(b), for Ti implants subjected to the various parameters in Table 1, the required wetting time increased with the storage time. As the storage time increased from 0 to $2 \mathrm{~h}$, the wetting time required also increased significantly from $2.7 \pm 0.4 \mathrm{~s}$ to about $5.7 \pm 0.1 \mathrm{~s}(P$ $<0.01)$. Any further increase in the storage time resulted in a slight increase in the mean wetting time, but not to a significant degree. After storage for more than $12 \mathrm{~h}$, the implant would no longer be wetted completely. This suggests that the hydrophilicity induced by the plasma treatment system would also degrade with increased storage time. Additionally, it can be seen that a long wetting time was always accompanied by large standard deviations, suggesting that the stability of the measured result decreased along with the decrease of hydrophilicity.

\section{Surface temperature}

To examine the optimal balance between the temperature safety and wettability of Ti implants during chair-side plasma treatment, the maximum temperature and wetting time of the treated implants were plotted as a function of various parameters. As shown in Fig. 6, as the treatment time increased from $7 \mathrm{~s}$ to $75 \mathrm{~s}$, the temperature of the implant increased slowly from $39.9 \pm 0.8^{\circ} \mathrm{C}$ to $55.3 \pm 0.9^{\circ} \mathrm{C}(P<0.01)$. An increase in the current of the $\mathrm{AC}$ source from $0.13 \mathrm{~A}$ to $0.21 \mathrm{~A}$ resulted in a sharp rise in the implant temperature from $39.0 \pm 2.6^{\circ} \mathrm{C}$ to $98.2 \pm 2.5^{\circ} \mathrm{C}(P<0.01)$. Once the current exceeded $0.15 \mathrm{~A}$, the implant temperature was difficult to maintain below $60^{\circ} \mathrm{C}$. This was because a more powerful input current releases more heat, while a long treatment time allows the implant to absorb enough heat until a thermal equilibrium is reached [24]. On the other hand, an increase in the argon flow rate from 1 SLM to 5 SLM lowered the temperature of the implant from $58.7 \pm 2.9^{\circ} \mathrm{C}$ to $45.1 \pm 2.1^{\circ} \mathrm{C}(P<0.01)$ due to promotion of heat exchange with the outside environment. Furthermore, addition of oxygen from $0 \%$ to $2.0 \%$ also caused a slight decline in the implant temperature from $50.9 \pm 1.4^{\circ} \mathrm{C}$ to $42.6 \pm 1.1^{\circ} \mathrm{C}(P<0.01)$ because ionization of oxygen requires consumption of extra plasma energy. More importantly, the wetting time was dependent only on the treatment time $(P<0.01)$ and was not affected by the input current of the AC source $(P=0.06)$, the argon flow rate $(P=0.71)$, or the oxygen content $(P=0.1)$, perhaps indicating that the implant surface was able to fully react with the plasma as long as the plasma was generated, irrespective of the plasma state. Therefore, 

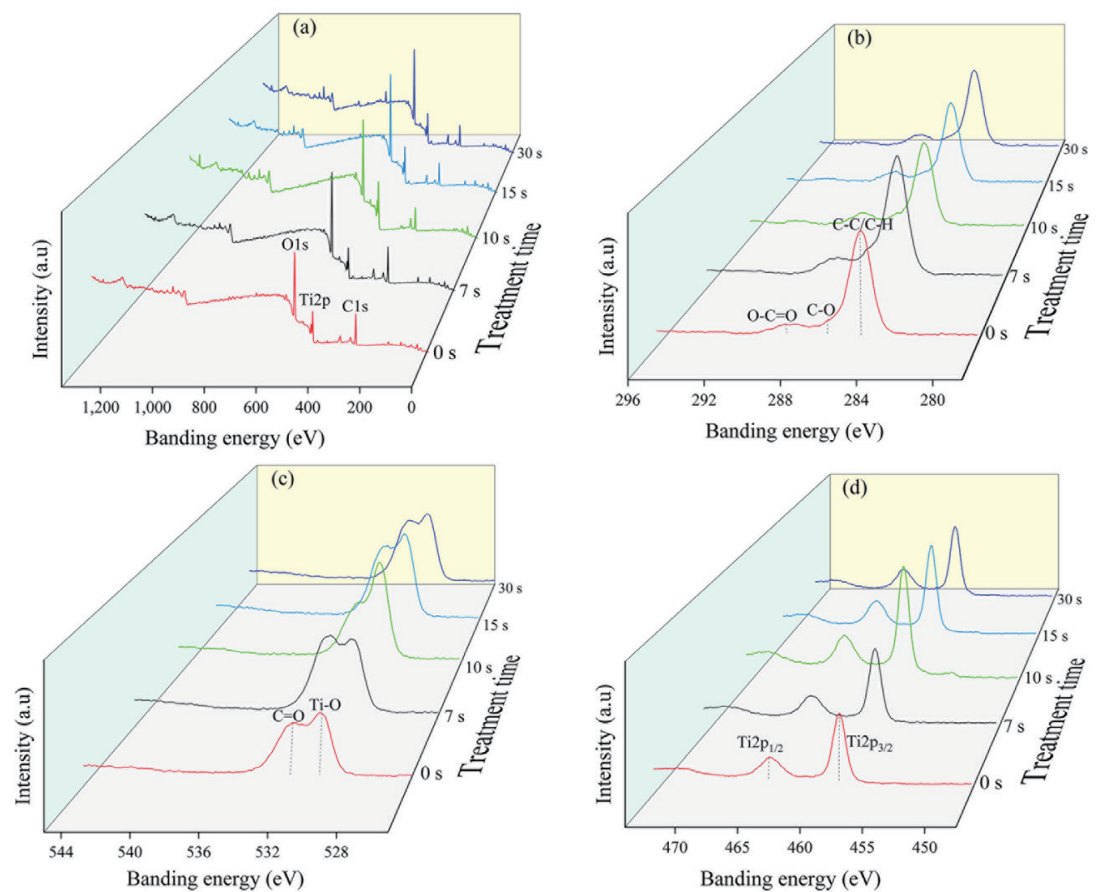

Fig. 7 XPS analysis of the Ti implant surface after different treatment time. Survey spectra (a), and high-resolution spectra of carbon (b), oxygen (c), titanium (d)

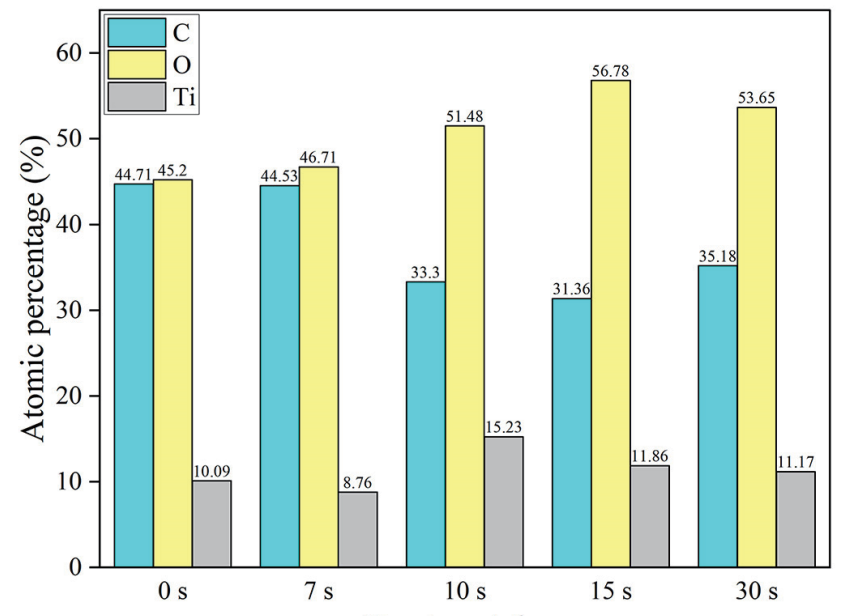

Treatment time

Fig. 8 The atomic percentages of carbon, oxygen and titanium on the implant surface after differen treatment times

selecting an AC current not exceeding $0.15 \mathrm{~A}$, an argon flow rate of 3 SLM and an oxygen content of $2 \%$ for hydrophilization for about $30 \mathrm{~s}$ yielded a highly hydrophilic implant at a temperature close to that of the human body.

\section{Changes in surface chemical composition}

In general, excited atoms and active radicals in a plasma treatment system can increase the surface energy of a Ti implant and change its surface chemical composition [14]. To explore the hydrophilization mechanism and chemical safety of plasma treatment, changes in the chemical composition of the implant surface during the treatment process were characterized by XPS. Figure 7(a) shows the XPS spectra of Ti implants after they had been treated for different times. It can be seen that the type of element on the implant surface after treatment had no significant impact. The peaks of $\mathrm{C} 1 \mathrm{~s}$ at $284.8 \mathrm{eV}$, Ti2p at $458.5 \mathrm{eV}$, and $\mathrm{O} 1 \mathrm{~s}$ at $530.4 \mathrm{eV}$ were clearly evident, and the plasma treatment did not introduce any harmful elements. Figure 7(b-d) show the high-resolution spectra of C1s, O1s, and Ti2p, respectively. After treatment, or as the treatment time increased, the peak intensity of elements may change according to changes in the chemical composition. By integrating the corresponding high-resolution spectra,

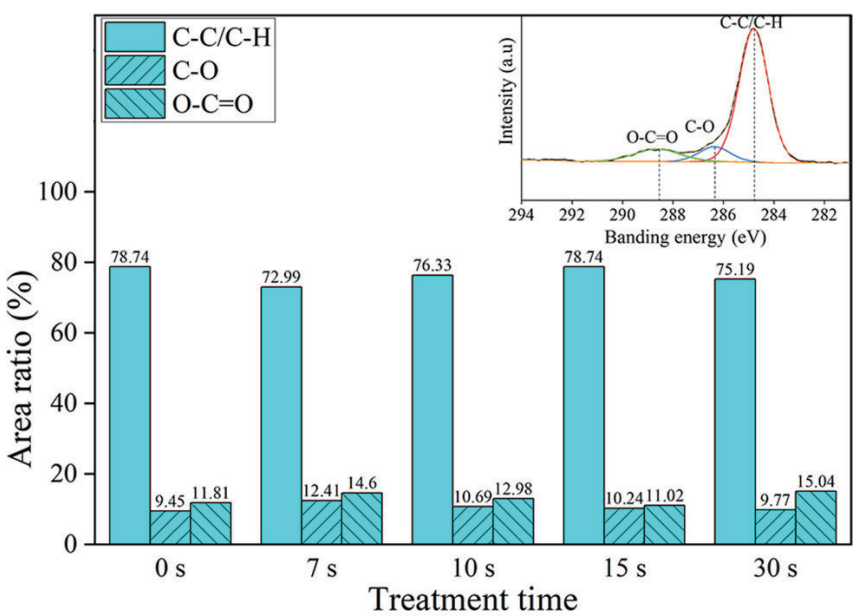

Fig. 9 Area ratios of carbon-related bond energy from XPS spectra of the implant surface after different treatment times. Inset shows the peak fitting curve of C-C/C-H at $284.8 \mathrm{eV}, \mathrm{C}-\mathrm{O}-\mathrm{C}$ at 286.4 $\mathrm{eV}$, and $\mathrm{O}-\mathrm{C}=\mathrm{O}$ at $288.4 \mathrm{eV}$.

the atomic ratios of carbon, oxygen, and titanium of the implant surface were calculated. As shown in Fig. 8, carbon and oxygen were the main components on the implant surface within a depth of $10 \mathrm{~nm}$. The titanium content, which could be regarded as a constant, was far less than that of carbon and oxygen. For the untreated implant, the $\mathrm{C} 1 \mathrm{~s}$ and $\mathrm{O} 1 \mathrm{~s}$ contents were roughly $45 \%$. When the plasma treatment lasted for $7 \mathrm{~s}$, the $\mathrm{C} 1 \mathrm{~s}$ and O1s contents showed no significant change. After treatment for $30 \mathrm{~s}$, the $\mathrm{C} 1 \mathrm{~s}$ content decreased to about $35 \%$. In contrast, the $\mathrm{O} 1 \mathrm{~s}$ content showed a slight increase relative to the fluctuation in the Ti2p content. These results indicate a reduction of carbon-containing compounds and introduction of more oxygen-containing groups during plasma treatment.

The Ti2p spectrum with a double-peak structure at $458.5 \mathrm{eV}$ and 464.5 $\mathrm{eV}$ appeared to confirm the presence of $\mathrm{TiO}_{2}$ [25]. The $\mathrm{C} 1 \mathrm{~s}$ spectrum was divisible into three components, including a C-C/C-H peak at $284.8 \mathrm{eV}$, a C-O-C peak at $286.4 \mathrm{eV}$, and a $\mathrm{O}-\mathrm{C}=\mathrm{O}$ peak at $288.4 \mathrm{eV}$, suggesting the presence of hydrocarbon contamination [26]. This could be expressed as $\mathrm{C}_{\mathrm{x}} \mathrm{H}_{\mathrm{y}} \mathrm{O}_{\mathrm{z}}$, in which the $\mathrm{C}-\mathrm{C} / \mathrm{C}-\mathrm{H}$ peak at $284.8 \mathrm{eV}$ was identified as the hydrocarbon, whereas the $\mathrm{C}-\mathrm{O}-\mathrm{C}$ peak at $286.4 \mathrm{eV}$ and the $\mathrm{O}-\mathrm{C}=\mathrm{O}$ peak at $288.4 \mathrm{eV}$ represented oxygen-containing functional groups such as ether and carboxyl [27]. It can be seen in Fig. 9 that the proportion of the three 


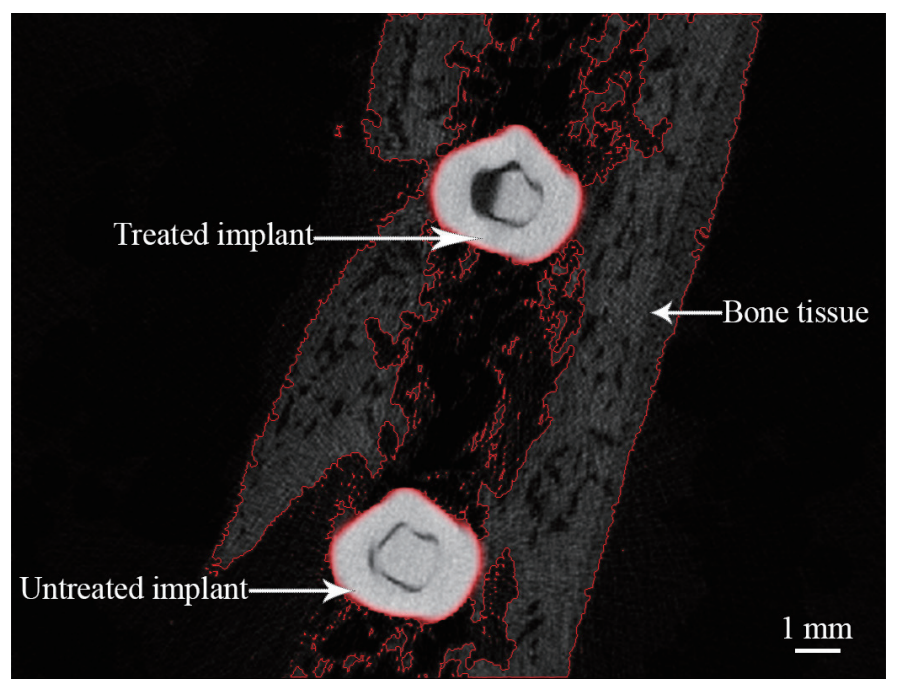

Fig. 10 Transverse micro CT image at the center of the treated and untreated implants after 4 weeks of implantation in vivo. Treatment parameters are detailed in Table 1 .

components in the $\mathrm{C} 1 \mathrm{~s}$ spectrum fluctuated within a small range and that the $\mathrm{C}-\mathrm{C} / \mathrm{C}-\mathrm{H}$ peak was always a dominator and fluctuated between $73 \%$ and $79 \%$. This suggests that the composition of the residual $\mathrm{C}_{\mathrm{x}} \mathrm{H}_{\mathrm{y}} \mathrm{O}_{\mathrm{z}}$ compound did not alter obviously during the plasma treatment.

\section{Osseointegration in vivo}

Figure 10 shows a transverse micro CT image of the center of untreated and treated implants after 4 weeks of implantation in vivo, where the white area represents the implant, the gray area with a red outline represents the bone tissue, and the black area indicates that there was no newly formed bone tissue. It can be seen that no adverse tissue reactions including injury or infection were observed in both the treated and untreated implants, confirming that the coaxial DBD plasma treatment system is safe and not harmful to the living body. Moreover, in comparison with the untreated implant, more newly formed bone tissue was observed around the treated implant, demonstrating that its hydrophilicity was able to promote bone osseointegration to some extent. Therefore, it can be concluded that implants subjected to cold plasma treatment are effective for promoting osseointegration and do not harm the living body.

\section{Discussion}

Generally, the hydrophilicity of material surfaces obtained using blasting and acid-etching is attributed to the formation of a micro-porous morphology [28]. Also, coating with hydroxyapatite, which has a high degree of biological activity, can change the hydrophilicity of a material [10]. In the case of ultraviolet irradiation and plasma treatment, the improvement of hydrophilicity is due to a change in surface free energy. Specifically, Williams and Lee et al. [14,19] considered that removal of hydrocarbon contamination yields high hydrophilicity after plasma treatment; whereas Chen and Steen et al. [26,29,30] considered that the introduction of oxygencontaining polar groups is the main factor for hydrophilicity enhancement, for the reason that a $100 \%$ hydrocarbon polymer material such as polyethylene could also obtain great hydrophilicity after plasma treatment.

In this study, a Ti dental implant was employed as a coaxial internal electrode, forming a DBD to generate plasma, thus facilitating rapid hydrophilization. It is necessary to explore the mechanism of hydrophilization achieved using this coaxial DBD plasma treatment system by conducting XPS to analyze the changes in composition of the implant surface. The results have confirmed that the improved hydrophilicity of an implant was due mainly to a decrease of hydrocarbon contamination. The removal of carbon contamination during the treatment process could be attributed to physical sputtering of ions and chemical reactivity of oxygen-containing active radicals [31,32]. Whether accelerated ion bombardment of the Ti implant surface can result in the desorption of carbon contamination depends on the potential difference between the plasma and the treated object [31]. Comparing with a plasma jet that provides only a low floating voltage not exceeding $20 \mathrm{~V}$, the coaxial DBD plasma treatment system can apply a high voltage in the order of kilovolts between the plasma and the implant to initiate physical sputtering [31], which can be confirmed by the fact that the pure argon plasma also improved the hydrophilicity of the implant (Fig. 6d). Moreover, the involvement of oxygen in the chemical reaction should also be recognized, where the active $\mathrm{O}$ atoms generated by the plasma break the C-C and C-O bonds of the $\mathrm{C}_{x} \mathrm{H}_{\mathrm{y}} \mathrm{O}_{z}$ compound and form more $\mathrm{C}=\mathrm{O}$ bonds, and ultimately volatile products such as $\mathrm{CO}_{2}$ [32]. However, neither physical sputtering of ions nor the chemical reaction would change the composition of the residual carbon contamination. Therefore, the XPS results showed a decrease in the $\mathrm{C} 1 \mathrm{~s}$ content (Fig. 8) but relatively constant levels of the $\mathrm{C}-\mathrm{C} / \mathrm{C}-\mathrm{H}, \mathrm{C}-\mathrm{O}-\mathrm{C}$, and $\mathrm{O}-\mathrm{C}=\mathrm{O}$ peaks (Fig. 9) after the plasma treatment. Here, removal of the $\mathrm{C}_{x} \mathrm{H}_{y} \mathrm{O}_{z}$ compound would have led to a decrease in the $\mathrm{O} 1 \mathrm{~s}$ content, but in fact it increased slightly after treatment (Fig. 8). This is because the oxygen content at the Ti implant surface existed mainly in the form of metal oxides, and the oxygen-containing plasma would have further oxidized this even if the untreated implant already had an oxide layer [33,34], which could also enhance the hydrophilicity $[26,29,30]$.

The Ti implant subjected to the coaxial DBD plasma treatment had shown high hydrophilicity characterized by a water contact angle close to $0^{\circ}$ and a wetting time of $3 \mathrm{~s}$. For the ultraviolet irradiation method proposed by Neobiotech, implants need a wetting time of at least $20 \mathrm{~s}$ [Young-goo $\mathrm{H}$ et al., Kr Patent 101972122B1, Apr 24, 2019]. In comparison to ultraviolet irradiation, plasma treatment confers better hydrophilicity on Ti implants. In addition, the coaxial DBD plasma treatment system can fully immerse a Ti implant in a plasma environment, and the entire implant can be treated homogeneously in a few seconds. While a plasma jet requires repeated scanning over the implant and a few minutes are needed to complete the hydrophilization $[15,18]$. Thus, the coaxial DBD plasma treatment system has a fast response and is easy to operate in a clinical setting.

Long-term storage is often considered to be detrimental to the hydrophilicity of an implant surface. Adhesion of external contamination and a decline in surface energy are responsible for the degradation of implant surface hydrophilicity under normal storage conditions. However, Lu et al. [11] have pointed out that degradation of hydrophilicity cannot be avoided even if an implant is stored in a sealed container. Thus the use of the coaxial DBD plasma treatment system for chair-side treatment in clinical operations would be an optimal way to avoid hydrophilicity degradation.

Dental implant osseointegration is susceptible to heat-induced damage. Once the temperature of bone tissue exceeds $60^{\circ} \mathrm{C}$ for $1 \mathrm{~min}$, the quality of osseointegration drops sharply [35]. For a chair-side plasma treatment system, it is necessary to keep the maximum temperature of an implant below $60^{\circ} \mathrm{C}$. The present experimental results suggest that an implant with high hydrophilicity can be obtained at a temperature close to that of the human body merely by setting the current at less than $0.15 \mathrm{~A}$, with an argon flow rate of 3 SLM, an oxygen content of $2 \%$, and a treatment time of $30 \mathrm{~s}$. Chemical safety should also be taken into account during plasma treatment because the introduction of harmful elements may cause corro- 
sion and release ions into the surrounding tissue, with unknown biological consequences [36]. The present XPS results indicated that the treated implant surface had no discernible harmful elements that might have been introduced by the coaxial DBD plasma treatment system. Therefore, the system should be safe enough for use on Ti dental implants in clinical operations.

In conclusion, it has been shown that plasma treatment of implants for less than $30 \mathrm{~s}$ can confer high hydrophilicity, as evidenced by a water contact angle close to $0^{\circ}$ and a wetting time of $3 \mathrm{~s}$. Enhancement of Ti implant surface hydrophilicity during plasma treatment is mainly attributable to a decrease of hydrocarbon contamination and the introduction of a denser oxide layer. The removal of hydrocarbon contamination is achieved by physical sputtering of ions and chemical reactivity of oxygen-containing active radicals. Furthermore, the temperature of the treated implant can be controlled to approximate that of the human body, and there is no introduction of any harmful elements during the plasma treatment. More importantly, improvement of osseointegration after 4 weeks of implantation was observed in vivo, which further verified the effectiveness and safety of the plasma treatment. Consequently, coaxial DBD plasma treatment appears to be a promising strategy for rapidly enhancing the surface hydrophilicity of dental implants in clinical operations without any need to consider the degradation of hydrophilicity caused by long-term storage.

Overall, the coaxial DBD plasma treatment system described here shows the excellent ability for improving the hydrophilicity of implant surfaces, and is effective and safe for clinical application. As a chair-side procedure, it should solve the problems related to the degradation of implant hydrophilicity by allowing practitioners to conduct hydrophilic treatment in clinical operations. To further verify its clinical effectiveness, more biological experiments will be conducted in the next study.

\section{Acknowledgments}

This work was financially supported by the National Natural Science Foundation of China (51875372, 52005353), the Strategic Cooperation Research Project between Sichuan University and Yibin City (2019CDYB7), the Technological innovation research and development project of Chengdu Science and Technology Bureau (2019-YF05-01328-SN), and the Miaozi Project in Science and Technology Innovation Program of Sichuan Province (20-YCG045).

\section{Conflict of interest}

The authors have no conflicts of interest to declare.

\section{References}

1. Derks J, Håkansson J, Wennström JL, Klinge B, Berglundh T (2015) Patient-reported outcomes of dental implant therapy in a large randomly selected sample. Clin Oral Implan Res 26, 586-591.

2. Toffoli A, Parisi L, Tatti R, Lorenzi A, Verucchi R, Manfredi E et al. (2020) Thermalinduced hydrophilicity enhancement of titanium dental implant surfaces. J Oral Sci 62 , $217-221$

3. Osman R, Swain M (2015) A critical review of dental implant materials with an emphasis on titanium versus zirconia. Materials 8, 932-958.

4. Rupp F, Scheideler L, Olshanska N, Wild M, Wieland M, Geis Gerstorfer J (2006) Enhancing surface free energy and hydrophilicity through chemical modification of microstructured titanium implant surfaces. J Biomed Mater Res A 76A, 323-334.

5. Hanawa T (2010) Biofunctionalization of titanium for dental implant. Jpn Dent Sci Rev 46, 93-101.

6. Lin L, Wang H, Ni M, Rui Y, Cheng T, Cheng C et al. (2014) Enhanced osteointegration of medical titanium implant with surface modifications in micro/nanoscale structures. J Orthop Translat 2, 35-42.

7. Sawase T, Jimbo R, Baba K, Shibata Y, Ikeda T, Atsuta M (2008) Photo-induced hydrophilicity enhances initial cell behavior and early bone apposition. Clin Oral Implan Res 19, 491-496.

8. Naujokat H, Harder S, Schulz LY, Wiltfang J, Flörke C, Y. Açil (2019) Surface condition- ing with cold argon plasma and its effect on the osseointegration of dental implants in miniature pigs. J Craniomaxillofac Surg 47, 484-490.

9. Velloso G, Moraschini V, Barboza EDSP (2019) Hydrophilic modification of sandblasted and acid-etched implants improves stability during early healing: a human double-blind randomized controlled trial. Int J Oral Maxillofac Surg 48, 684-690.

10. Visentin F, Habra NEl, Fabrizio M, Brianese N, Gerbasi R, Nodari L et al. (2019) TiO2-HA bi-layer coatings for improving the bioactivity and service-life of Ti dental implants. Surf Coat Technol 378, 125049.

11. Haibin L, Lei W, Xueyang Z, Mingdeng R, Zehong G, Lei Z (2013) Effects of hydrocarbons contamination on initial responses of osteoblast-like cells on acid-etched titanium surface. Rare Metal Mat Eng 42,1558-1562.

12. Duske K, Jablonowski L, Koban I, Matthes R, Holtfreter B, Sckell A et al. (2015) Cold atmospheric plasma in combination with mechanical treatment improves osteoblast growth on biofilm covered titanium discs. Biomaterials 52, 327-334.

13. Mui TSM, Silva LLG, Prysiazhnyi V, Kostov KG (2017) Surface modification of aluminium alloys by atmospheric pressure plasma treatments for enhancement of their adhesion properties. Surf Coat Technol 312, 32-36.

14. Williams DF, Kellar EJC, Jesson DA, Watts JF (2017) Surface analysis of 316 stainless steel treated with cold atmospheric plasma. Appl Surf Sci 403, 240-247.

15. Han I, Vagaska B, Seo HJ, Kang JK, Kwon B, Lee MH et al. (2012) Promoted cell and material interaction on atmospheric pressure plasma treated titanium. Appl Surf Sci 258 , 4718-4723.

16. Mui TSM, Mota RP, Quade A, Luis, Rogério De Oliveira Hein KGK (2018) Uniform surface modification of polyethylene terephthalate (PET) by atmospheric pressure plasma jet with a horn-like nozzle. Surf Coat Technol 352, 338-347.

17. Chu Paul K (2007) Enhancement of surface properties of biomaterials using plasma-based technologies. Surf Coat Technol 201, 8076-8082.

18. Duske K, Koban I, Kindel E, Schröder K, Nebe B, Holtfreter B et al. (2012) Atmospheric plasma enhances wettability and cell spreading on dental implant metals. J Clin Periodontol $39,400-407$.

19. Lee J, Jeong W, Seo S, Kim H, Kim K, Choi E et al. (2017) Non-thermal atmospheric pressure plasma functionalized dental implant for enhancement of bacterial resistance and osseointegration. Dent Mater 33, 257-270.

20. Guastaldi FPS, Yoo D, Marin C, Jimbo R, Tovar N, Zanetta-Barbosa D et al. (2013) Plasma treatment maintains surface energy of the implant surface and enhances osseointegration. Int J Biomater 2013, 354125.

21. Pizzorni M, Gambaro C, Lertora E, Mandolfino C (2018) Effect of process gases in vacuum plasma treatment on adhesion properties of titanium alloy substrates. Int J Adhes Adhes 86 , 113-122.

22. Wang F, Li C, Zhang S, Liu H (2020) Tantalum coated on titanium dioxide nanotubes by plasma spraying enhances cytocompatibility for dental implants. Surf Coat Technol 382, 125161 .

23. Faul F, Erdfelder E, Buchner A, Lang A-G (2009) Statistical power analyses using G*Power 3.1: Tests for correlation and regression analyses. Behav Res Methods 41, 1149-1160.

24. Kim K, Kim G, Hong YC, Yang SS (2010) A cold micro plasma jet device suitable for bio-medical applications. Microelectron Eng 87, 1177-1180.

25. Acciari HA, Palma DPS, Codaro EN, Zhou Q, Wang J, Ling Y et al. (2019) Surface modifications by both anodic oxidation and ion beam implantation on electropolished titanium substrates. Appl Surf Sci 487, 1111-1120.

26. Shao X, Zhang G, Zhan J, Xu G (2011) Research on surface modification of polytetrafluoroethylene coupled with argon dielectric barrier discharge plasma jet characteristics. IEEE Trans Plasma Sci 39, 3095-3102.

27. Boehm HP (2002) Surface oxides on carbon and their analysis: a critical assessment. Carbon 40, 145-149.

28. Reshadi F, Khorasani S, Faraji G (2020) Surface characterization of nanostructured commercially pure titanium modified by blast and acid-etching for implant applications. Proc Inst Mech Eng J J Eng Tribol 234, 414-423.

29. Steen ML, Jordan AC, Fisher ER (2002) Hydrophilic modification of polymeric membranes by low temperature $\mathrm{H} 2 \mathrm{O}$ plasma treatment. J Membrane Sci 204, 341-357.

30. Cheng C, Liye Z, Zhan R (2006) Surface modification of polymer fibre by the new atmospheric pressure cold plasma jet. Surf Coat Technol 200, 6659-6665.

31. Belkind A, Gershman S (2008) Plasma cleaning of surface. Vac Technol Coat 2008, 1-11.

32. Zhang Z, Ye Z, Wang Z, Gou F, Shen B, Wu A et al. (2019) The mechanism study of mixed $\mathrm{Ar} / \mathrm{O} 2$ plasma-cleaning treatment on niobium surface for work function improvement. Appl Surf Sci 475, 143-150.

33. Fuchs $\mathbf{P}$ (2009) Low-pressure plasma cleaning of Au and PtIr noble metal surfaces. Appl Surf Sci 256, 1382-1390.

34. Henningsen A, Smeets R, Hartjen P, Heinrich O, Heuberger R, Heiland M et al. (2018) Photofunctionalization and non-thermal plasma activation of titanium surfaces. Clin Oral Investig 22, 1045-1054

35. Trisi P, Berardini M, Falco A, Vulpiani MP (2015) Effect of temperature on the dental implant osseointegration development in low-density bone: an in vivo histological evaluation. Implant Dent 24, 96-100.

36. Dias FJ, Fuentes R, Navarro P, Weber B, Borie E (2020) Assessment of the chemical composition in different dental implant types: an analysis through EDX system. Coatings 10 , 882. 